\title{
Estudo cinético da secagem da casca de jabuticaba (Myrciaria Cauliflora Berg) utilizando modelos empíricos e semi-empíricos
}

\author{
Kinetic study of the drying of the bark of jabuticaba (Myrciaria Cauliflora Berg) using empirical \\ and semi-empirical models \\ Estudio cinético del secado de la corteza de jabuticaba (Myrciaria Cauliflora Berg) utilizando \\ modelos empíricos y semi-empíricos
}

\author{
Helton Gomes Alves \\ ORCID: https://orcid.org/0000-0001-6456-9261 \\ Universidade Federal do Rio Grande do Norte, Brasil \\ E-mail:heltongomes225@gmail.com \\ Raphael Lucas Jacinto Almeida \\ ORCID: https://orcid.org/0000-0001-7232-2373 \\ Universidade Federal do Rio Grande do Norte, Brasil \\ E-mail:raphaelqindustrial@gmail.com \\ Didiane Saraiva da Silva \\ ORCID: https://orcid.org/0000-0002-2742-612X \\ Universidade Estadual da Paraíba, Brasil \\ E-mail:didiane_saraiva@hotmail.com \\ Vitória de Andrade Freire \\ ORCID: https://orcid.org/0000-0001-8861-2324 \\ Universidade Federal de Campina Grande, Brasil \\ E-mail:vitoriaqi114@gmail.com \\ Valmara Silva Araújo \\ ORCID: https://orcid.org/0000-0001-9538-6257 \\ Universidade Estadual da Paraíba, Brasil \\ E-mail: valmarasilva16@hotmail.com \\ Ângela Maria Santiago \\ ORCID: https://orcid.org/0000-0001-7108-6890 \\ Universidade Estadual da Paraíba, Brasil \\ E-mail: angelamariasantiago01@gmail.com \\ Líbia de Sousa Conrado \\ ORCID: https://orcid.org/0000-0001-6384-0793 \\ Universidade Federal de Campina Grande, Brasil \\ E-mail: libiaconrado@yahoo.com.br \\ Pablícia Oliveira Galdino \\ ORCID: https://orcid.org/0000-0001-6996-0550 \\ Universidade Estadual da Paraíba, Brasil \\ E-mail: pabliciagaldino4@gmail.com \\ Marcello Maia de Almeida \\ ORCID: https://orcid.org/0000-0001-7889-126X \\ Universidade Estadual da Paraíba, Brasil \\ E-mail: marcello_maia2000@yahoo.com.br \\ Wanda Izabel Monteiro de Lima Marsiglia \\ ORCID: https://orcid.org/0000-0002-1137-576X \\ Universidade Estadual da Paraíba, Brasil \\ E-mail: wandaequepb@gmail.com.br
}

\begin{abstract}
Resumo
A Jabuticabeira (Myrciaria cauliflora) é uma árvore frutífera nativa brasileira, pertencente à família Myrtaceae, seus frutos possuem grande valor nutricional. As cascas de jabuticaba são uma fonte de antocianinas, os quais são poderosos compostos antioxidantes e apresentam atividade antimicrobiana. Contudo, as cascas de jabuticaba são geralmente descartadas, o que além de gerar resíduos poluentes, promove o desperdício de nutrientes e compostos potencialmente bioativos presentes nessa porção. Assim, com o objetivo de minimizar as alterações e perdas de determinados compostos bioativos, e consequentemente indicar o potencial do aproveitamento do resíduo em escala industrial, o presente trabalho tem com objetivo avaliar a cinética de secagem da casca de jabuticaba e ajustar modelos matemáticos aos dados experimentais. Os frutos foram adquiridos no comércio de Campina Grande/PB, selecionados
\end{abstract}


de acordo com o grau de maturação apropriada para o processamento e ausência de danos físicos (injúrias), lavados e higienizados com solução de hipoclorito de sódio, em seguida foram despolpados manualmente para separação das frações sólidas, polpa, casca e sementes. A secagem foi realizada em uma estufa de circulação de ar forçado, na faixa de temperaturas 45,50 e $55^{\circ} \mathrm{C}$. Os dados experimentais da secagem foram ajustados aos modelos de Page, Midili e Henderson e Pabis. A cinética das cascas de Jabuticaba mostrou que a temperatura influenciou no processo de secagem, e constatou-se que a constante de secagem " $k$ " aumentou com a sua elevação. Ademais, o modelo de Midili apresentou um melhor ajuste comparado aos demais métodos.

Palavras-chave: Resíduo agroindustrial; Alimento funcional; Processo de secagem; Modelagem matemática.

\section{Abstract}

The Jabuticabeira (Myrciaria cauliflora) is a Brazilian native fruit tree, belonging to the family Myrtaceae, its fruits have great nutritional value. Jabuticaba shells are a source of anthocyanins, which are powerful antioxidant compounds and have antimicrobial activity. However, jabuticaba peels are generally discarded, which in addition to generating polluting waste, promotes the waste of nutrients and potentially bioactive compounds present in that portion. Thus, in order to minimize the changes and losses of certain bioactive compounds, and consequently indicate the potential for using the waste on an industrial scale, the present work aims to evaluate the drying kinetics of jabuticaba bark and adjust mathematical models to experimental data. The fruits were purchased at the Campina Grande - PB, selected according to the degree of maturity appropriate for processing and absence of physical damage (injuries), washed and sanitized with sodium hypochlorite solution, then they were manually pulped to separate the solid fractions, pulp, peel and seeds. The drying was carried out in a forced air circulation oven, at temperatures range 45, 50 and $55^{\circ} \mathrm{C}$. The experimental drying data were adjusted to the Page, Midili and Henderson and Pabis models. The kinetics of the Jabuticaba shells showed that the temperature influenced the drying process, and it was found that the drying constant " $k$ " increased with its elevation. In addition, the Midili model showed a better fit compared to the other methods.
\end{abstract}

Keywords: Agro-industrial waste; Functional food; Drying process; Mathematical modeling.

\title{
Resumen
}

La Jabuticabeira (Myrciaria cauliflora), perteneciente a la familia Myrtaceae, sus frutos tienen un gran valor nutricional. Las cáscaras de jaboticaba son una fuente de antocianinas, que son potentes compuestos antioxidantes y tienen actividad antimicrobiana. Todavía, Las cáscara de jabuticaba generalmente se descartan, que además de generar residuos contaminantes, promueve el desperdicio de nutrientes y compuestos potencialmente bioactivos presentes en esa porción. Así, com o objetivo de minimizar as alterações e perdas de determinados compostos bioativos, y, en consecuencia, indica el potencial de uso de los residuos a escala industrial, El presente trabajo tiene como objetivo evaluar la cinética de secado de la corteza de jabuticaba y ajustar los modelos matemáticos a los datos experimentales. Las frutas se compraron en Campina Grande - PB, seleccionados de acuerdo con el grado de madurez apropiado para el procesamiento y la ausencia de daño físico (lesiones), lavado y desinfectado con solución de hipoclorito de sodio, luego se abierto manualmente para separar las fracciones sólidas, pulpa, cáscara y semillas. El secado se realizó en un horno de circulación de aire forzado, a temperaturas de 45,50 y $55^{\circ} \mathrm{C}$ y una velocidad de $1.0 \mathrm{~m} / \mathrm{s}$. Los datos de secado experimental se ajustaron a los modelos de Page, Midili y Henderson y Pabis. La cinética de las conchas de Jabuticaba mostró que la temperatura influyó en el proceso de secado y se encontró que la constante de secado "k" aumentaba con su elevación. Además, el modelo Midili mostró un mejor ajuste en comparación con los otros métodos.

Palabras clave: Residuos agroindustriales; Comida funcional; Proceso de secado; Modelo matemático.

\section{Introdução}

A jabuticabeira (Plinia sp.) pertence à família Myrtaceae e é encontrada em extensa faixa no país, do Rio Grande do Sul até o Pará, sendo que as maiores produções se concentram nos estados de São Paulo, Rio de Janeiro, Minas Gerais e Espírito Santo (Lima et al., 2008; Ferreira et al., 2012;). Dentre as espécies, destacam-se a Myrciaria cauliflora (DC) Berg (jabuticaba paulista) e a Myrciaria jabuticaba (Vell) Berg (jabuticaba Sabará) (Donadio, 2000; Ascheri et al., 2006).

A jabuticaba é de grande interesse dos produtores rurais, devido à sua alta produtividade, rusticidade e aproveitamento sob diversas formas (Ferreira et al., 2012). Seus frutos possuem casca de cor roxa quase preta, formato esférico de até $3,5 \mathrm{~cm}$ de diâmetro, polpa esbranquiçada mucilaginosa (Figura 1), translúcidas, agridoce, leve acidez muito saborosa, apresenta geralmente poucas sementes, podendo apresentar até 4 sementes. (Lima et al., 2008; Teixeira, 2011; Cipriano, 2012).

A jabuticaba pode ser consumida in natura ou processada, sendo utilizados na fabricação de vinhos, geleia, sucos, licor, aguardente. Além disso, os frutos podem ser aproveitados pela indústria farmacêutica e alimentícia, devido a seu alto teor 
de substâncias antioxidantes. O uso das jabuticabeiras como planta ornamental também é indicado, pela exuberância e beleza da florada e frutificação. (Manica, 2000; Citadin, 2010; Cipriano, 2012).

Figura 1 - Fruto da Jabuticabeira (Plinia sp.) oriunda do comércio do município de Campina Grande.

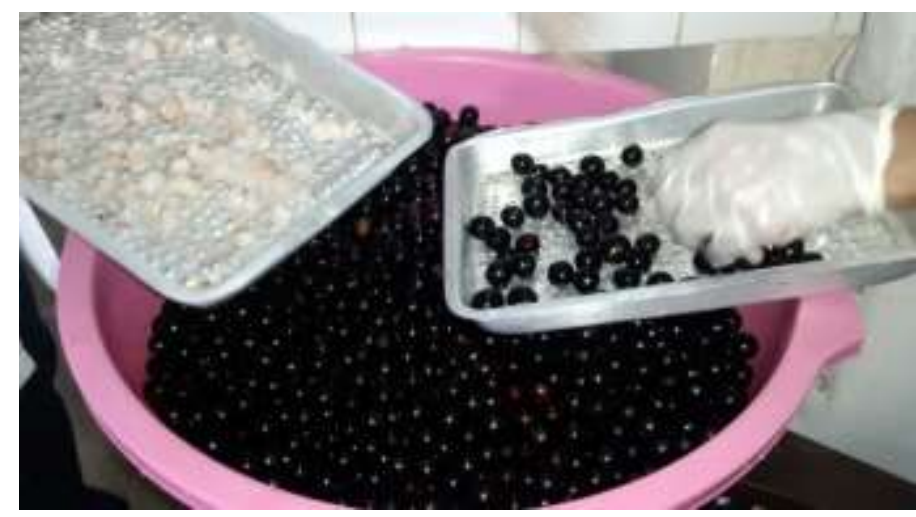

Fonte: Autores.

De acordo com Ferreira et al. (2012), a jabuticaba possui grande valor nutricional, apresentando teores significativos de fibras $(2,3 \mathrm{~g} / 100 \mathrm{~g})$, potássio $(130 \mathrm{mg} / 100 \mathrm{~g})$ e magnésio $(18 \mathrm{mg} / 100 \mathrm{~g})$. As cascas e sementes da jabuticaba, geralmente desprezadas, representam juntas, aproximadamente, 50\% do fruto (Lima et al., 2008; Sousa \& Correia, 2010). A casca da jabuticaba tem propriedades adstringentes, eficazes contra diarreia e irritações da pele, são ricas em compostos fenólicos, pectina e açúcares podendo ser utilizadas além de antioxidantes, como corantes alimentares naturais (Alezandro et al., 2013). Pesquisas apontam que chás e sucos feitos com a casca de jabuticaba podem auxiliar no tratamento de problemas de saúde como alergias, fragilidade capilar, amigdalite, infecções intestinais, varizes, erisipela, asma, entre outros. (Brunini, 2004; Lima et al., 2008; Silva, 2012; Santiago et al., 2012; Abreu et al., 2013).

Porém, o fruto possui uma alta perecibilidade, devido seu alto teor de água e açucares a jabuticaba apresenta um período curto de comercialização após a colheita, resultado da intensa fermentação, deterioração e intensa perda de umidade (Ascheri et al., 2006; Nunes et al, 2014). Assim, faz se necessário à utilização de métodos de conservação a fim de que o alimento se torne estável à deterioração química e microbiana (Machado, 2014), dentre elas a técnica de secagem pode acarretar um melhor aproveitamento dessas frações, agregando maior valor comercial ao fruto.

Dentre os principais privilégios apresentados pela secagem de frutas está um maior tempo de vida de prateleira, a concentração e a conservação dos nutrientes. Outrossim, a permanência e a longevidade do sabor, devido a minimização da proliferação de micro-organismos. (Machado, 2015).

A secagem é atualmente empregada não apenas com o objetivo de conservação dos alimentos, mas também para elaboração de produtos diferenciados, como por exemplo, as massas, biscoitos, farinhas, iogurtes, cremes, sorvetes entre outros (Fioreze, 2004; Silva et al., 2010; Gurak, et al., 2014; Almeida et al., 2019; Silva et al., 2020; Garcia et al., 2020).

No entanto, de acordo com Almeida et al. (2019) os subprodutos da indústria de frutas e vegetais são constituídos principalmente por: cascas, frações de bagaço e sementes, que podem ser uma boa fonte de compostos bioativos, além de conterem na sua composição carboidratos, fibras alimentares, compostos aromatizantes e fotoquímicos. Normalmente, as cascas do fruto da Jabuticaba são descartadas, correspondendo cerca de $30 \%$ a $43 \%$ do fruto, gerando grande quantidade de resíduo (Alezandro et al., 2013). Os extratos da casca de jabuticaba contêm elevado teor de compostos bioativos (antioxidantes, antocianinas, compostos fenólicos e taninos), que proporcionam benefícios relativos à saúde para o 
consumidor, contribui para parâmetros de controle relacionados à inflamação, perfil lipídico e glicêmico, estresse oxidativo e radicais livres (Abreu \& Ferreira, 2013; Garcia et al., 2020).

A aplicação dos modelos matemáticos confiáveis permite prever o comportamento dos diversos fenômenos que ocorrem durante o processo de secagem, o que implica na redução do custo operacional (Dionello et al., 2009). O estudo e a modelagem matemática da cinética de secagem têm despertado o interesse de vários pesquisadores para os mais diferentes produtos. Na literatura, diversos modelos matemáticos têm sido utilizados para descrever a cinética de secagem em camada fina para produtos agrícolas: secagem de mangaba (Medeiros, 2007), secagem de fatia do abacaxi (Alexandre et al., 2009), secagem dos frutos de lichia (Janjai et al., 2010); desidratação da polpa da pimenta cumari (Reis et al., 2011), secagem da semente de Cambre (Faria et al., 2012), secagem da casca da jabuticaba (Nunes et al., 2014 e Marsiglia et al., 2021), desidratação dos frutos do amendoim (Araújo et al., 2017), Secagem de café (Tosta et al., 2020) e Secagem de nozes ( Silva et al., 2020).

Neste contexto, com o intuito de minimizar as alterações e perdas de determinados compostos bioativos durante o processo de secagem, e consequentemente indicar o potencial do aproveitamento do resíduo em escala industrial, o presente trabalho tem como objetivo avaliar a cinética de secagem da casca de jabuticaba e ajustar modelos matemáticos, que represente de maneira clara e satisfatória todos os dados requeridos experimentalmente, selecionando o qual melhor descreve o fenômeno.

\section{Metodologia}

Os processos de secagem foram realizados no Laboratório de Engenharia Bioquímica (LEB) vinculado a Unidade acadêmica de Engenharia Química, da Universidade Federal de Campina Grande, PB.

A matéria prima utilizada foram os frutos da Jabuticabeira (Myrciaria cauliflora Berg), adquiridos no comércio do município de Campina Grande - PB. Os frutos foram selecionados no laboratório de acordo com o grau de maturação apropriada para o processamento e ausência de danos físicos (injúrias), em seguida foram submetidos à lavagem em água corrente, sanitizados em solução de Hipoclorito de sódio (1,5mL a cada litro), enxaguados e despolpados manualmente para separação das frações sólidas, a polpa, a casca e as sementes.

A secagem foi realizada em uma estufa de circulação de ar forçado com temperatura controlada, às cascas foram adicionadas em bandejas de alumínio circulares, contendo cada bandeja $100 \mathrm{~g}$ de cascas, a qual foi uniformemente espalhada. As curvas foram determinadas com três níveis de temperatura $\left(45,50\right.$ e $\left.55^{\circ} \mathrm{C}\right)$. A pesagem das amostras para determinar à perda de peso foi realizada através de uma balança semi analítica com intervalos de tempo de 10 e 10 min durante os primeiros 100 min, de 20 em 20min para os 160 min seguintes, de $30 \mathrm{em} 30$ para os 300min seguintes e de 60 em 60 min até peso constante.

Os dados experimentais foram expressos na forma de razão umidade, obtidos pela conversão dos dados referentes à perda de água no parâmetro adimensional $\left(\mathrm{X}^{*}\right)$ :

$$
X^{*}=\frac{\left(X(t)-X_{e q}\right)}{\left(X_{i}-X_{e q}\right)}
$$

onde, $\mathrm{X}(\mathrm{t})$ corresponde a umidade em base seca em função do tempo (bs), $\mathrm{X}_{\mathrm{eq}}$ é a umidade de equilíbrio (bs) e $\mathrm{X}_{\mathrm{i}}$ é umidade inicial (bs). 
A umidade de equilíbrio (bs) nas temperaturas em estudo foi determinada através da extrapolação das curvas de taxa de secagem, derivada da umidade base seca em relação ao tempo versus umidade base seca média $\left(\frac{d X}{d t}\right.$ versus $\left.\mathrm{X}_{\mathrm{m}}\right)$ em cada temperatura.

Os valores experimentais de razão de umidade para temperatura foram simulados com três equações empíricas e semiempíricas frequentemente utilizadas para a representação da secagem de produtos agrícolas. Através de regressão não linear, os modelos matemáticos de Page, Midili e Henderson \& Pabis, descritos na Tabela 1, foram ajustados às curvas experimentais de secagem utilizando-se o programa computacional Statistica® versão 7.0. Para se determinar o melhor ajuste de cada modelo aos dados experimentais foram observados a magnitude dos coeficientes de determinação $\left(\mathrm{R}^{2}\right)$.

Tabela 1 - Modelos de Regressão não-linear utilizados para predizer o fenômeno de Secagem da casca de Jabuticaba.

\begin{tabular}{cl}
\hline Modelo & Equação \\
\hline Page & $U^{*}=\exp \left(-\kappa t^{n}\right)$ \\
Midili & $U^{*}=a \exp \left(-\kappa t^{n}\right)+b t$ \\
Henderson \& Pabis & $U^{*}=a \exp (-\kappa t)$ \\
\hline
\end{tabular}

$\mathrm{U}^{*}$ - razão de unidade (bs); t - tempo (min); a, k, b e n - parâmetros dos modelos. Fonte: Autores.

\section{Resultados e Discussão}

Os valores de umidade de equilíbrio (Xeq), obtidas para cada temperatura aplicada foram de 0,1043, 0,1024 e 0,1006 (bs) para as temperaturas de 45,50 e $55^{\circ} \mathrm{C}$, respectivamente. Desta forma, pode se observar que a umidade de equilíbrio foi inversamente proporcional ao aumento da temperatura de secagem, comportamento já evidenciado por Silva et al. (1979) ao estudar a cinética de secagem da semente do cacau, por Alexandre et al. (2009) ao estudar o processo de secagem de abacaxi em fatias sem talo e também por Nunes et al. (2014) ao estudar o processo de desidratação da polpa da jabuticaba.

Na Figura 2, estão representadas as curvas de secagem da casca da Jabuticaba, nas temperaturas de 45,50 e $55^{\circ} \mathrm{C}$, na forma de adimensional (razão de umidade) em função do tempo. 
Figura 2 - Curvas cinéticas da secagem da casca da Jabuticaba nas temperaturas de 45,50 e $55^{\circ} \mathrm{C}$.

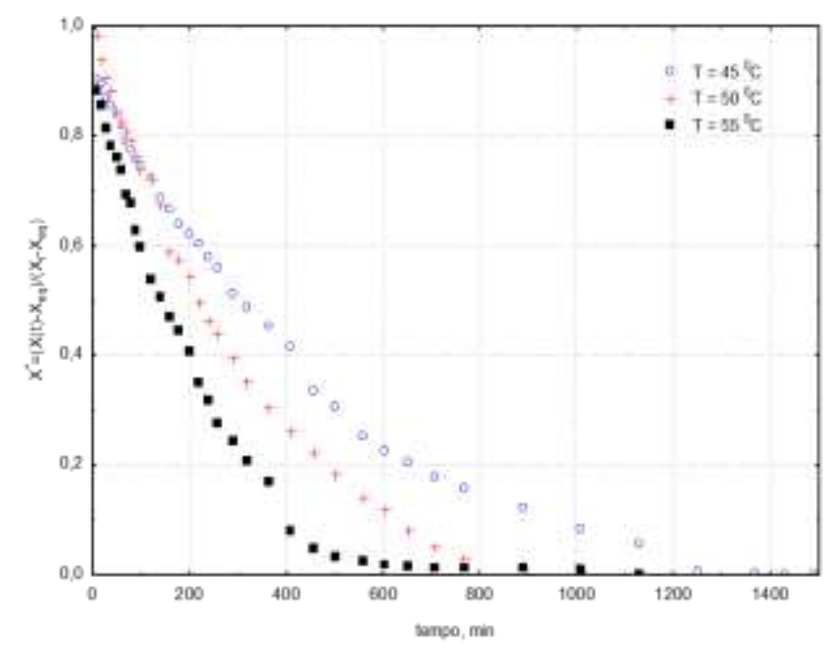

Fonte: Autores.

Pode-se observar que as curvas da cinética de secagem apresentam um período de taxa decrescente. Tem-se perda de massa mais rápido no início da secagem, com a redução gradativa de acordo com o avanço do processo, o aumento da temperatura do ar de secagem promoveu uma maior taxa de remoção de água do produto, este comportamento evidencia a influência da temperatura do ar de secagem sob a cinética de secagem de alimentos, como observado por Gouveia et al. (2002), Akpinar (2006), Alexandre et al. (2009), Santos et al. (2012), Nunes et al. (2014), Machado (2015), Silva et al., (2020), Garcia et al., (2020) e Marsiglia et al., (2021).

A Figura 3 apresenta as curvas dos modelos de Page, Midili e Henderson \& Pabis ajustados aos dados experimentais. E na tabela 2 estão apresentados os valores dos parâmetros obtidos no ajuste dos modelos matemáticos aos dados experimentais do processo de secagem das cascas da jabuticaba, bem como os coeficientes de determinação $\left(\mathrm{R}^{2}\right)$, para as temperaturas de 45,50 e $55^{\circ} \mathrm{C}$. 
Figura 3 - Curvas da cinética de secagem da casca da jabutica ajustadas para os modelos em de Page, Midili e Henderson \& Pabis. (a) $\mathrm{T}=45$; (b) $\mathrm{T}=50$ e (c) $\mathrm{T}=55^{\circ} \mathrm{C}$.

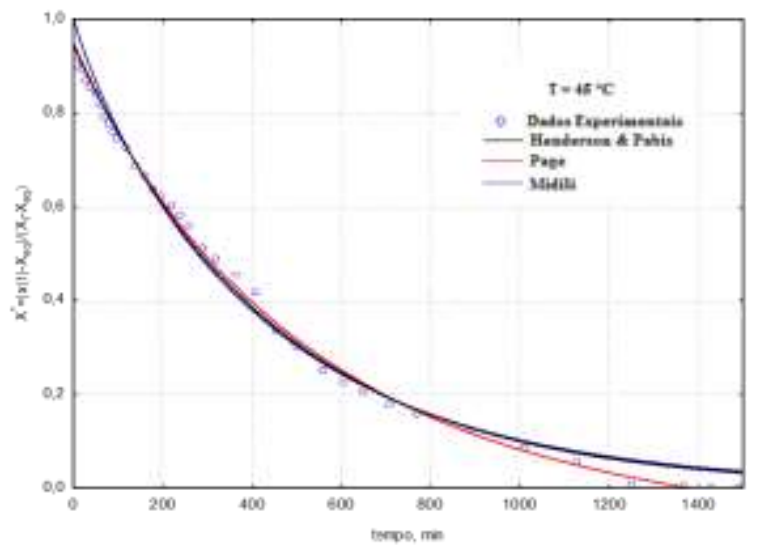

(a)

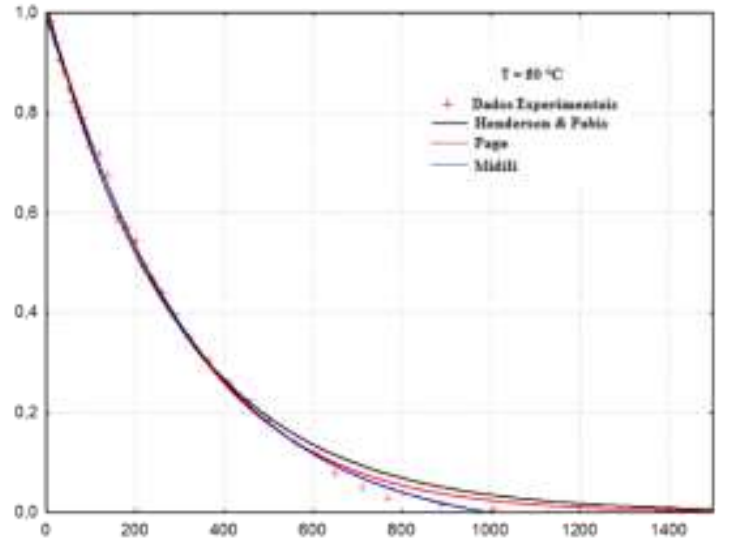

(b)

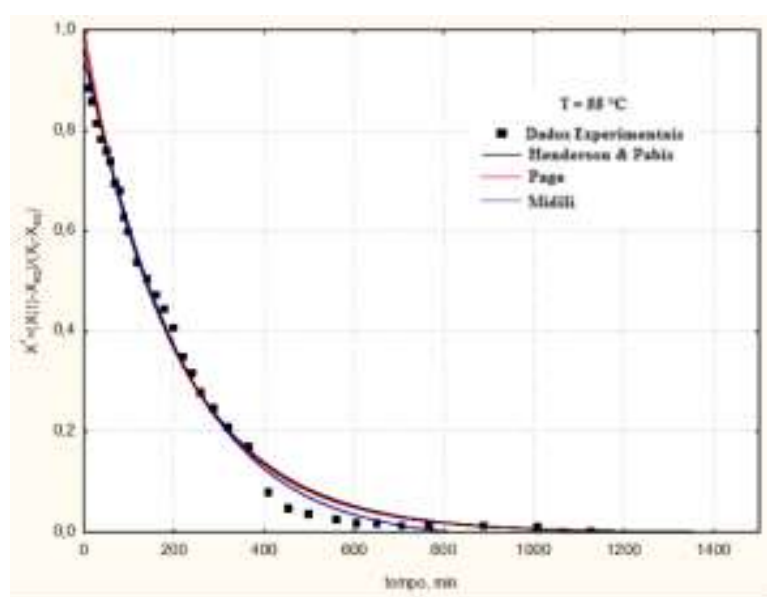

(c)

Fonte: Autores.

Tabela 2 - Parâmetros e coeficientes de determinação dos modelos das curvas de secagem das cascsa da Jabuticaba ajustados para todas as temperaturas.

\begin{tabular}{|c|c|c|c|c|c|c|}
\hline \multirow[b]{2}{*}{ Modelo } & \multirow[b]{2}{*}{ Temperatura $\left({ }^{\circ} \mathbf{C}\right)$} & \multicolumn{4}{|c|}{ Parâmetros } & \multirow[b]{2}{*}{$\mathbf{R}^{2}$} \\
\hline & & $\mathbf{K}_{1}$ & $\mathbf{N}$ & - & - & \\
\hline \multirow[b]{3}{*}{ Page } & 45 & 0,0037 & 1,0998 & - & & 0,9901 \\
\hline & 50 & 0,0048 & 0,9905 & - & - & 0,9978 \\
\hline & 55 & 0,0053 & 0,9293 & - & - & 0,9922 \\
\hline \multirow[b]{4}{*}{ Midili } & Temperatura $\left({ }^{\circ} \mathrm{C}\right)$ & $\mathbf{K}_{2}$ & $\mathbf{N}$ & $\mathbf{a}$ & $\mathbf{b}$ & $\mathbf{R}^{2}$ \\
\hline & 45 & 0,0024 & 1,0970 & 0,9427 & $-0,0004$ & 0,9969 \\
\hline & 50 & 0,0025 & 0,9965 & 0,9398 & $-0,0008$ & 0,9988 \\
\hline & 55 & 0,0027 & 0,9746 & 0,9375 & $-0,0015$ & 0,9952 \\
\hline \multirow{4}{*}{$\begin{array}{c}\text { Henderson } \\
\text { \& Pabis }\end{array}$} & Temperatura $\left({ }^{\circ} \mathrm{C}\right)$ & $\mathbf{K}_{3}$ & $\mathbf{a}$ & - & - & $\mathbf{R}^{2}$ \\
\hline & 45 & 0,0023 & 0,9488 & - & - & 0,9942 \\
\hline & 50 & 0,0034 & 1,0021 & - & - & 0,9959 \\
\hline & 55 & 0,0049 & 0,9695 & - & - & 0,9935 \\
\hline
\end{tabular}

Fonte: Autores. 


\section{Conclusão}

As equações matemáticas obtidas, nas condições utilizadas no presente trabalho, foram capazes de predizer o comportamento da cinética do processo de secagem das cascas de jabuticabas. No entanto, o modelo de Midilli se destacou dos demais por apresentar o maior valor da correlação $\mathrm{R}^{2}$. De acordo com a análise da cinética, também foi possível observar que a temperatura influenciou no processo de desidratação, e a constante de secagem " $k$ ” aumentou com a sua elevação.

\section{Referências}

Abreu, H., \& Ferreira, S. M. R. (2013) Extração e encapsulação de antocianinas de Jabuticaba (Myrciariacauliflora), Açaí (Euterpe oleracea) e Uva Isabel (Vitis labrusca). In: $8^{\circ}$ EETCG - Encontro de Engenharia e Tecnologia dos Campos Gerais, 2013.

Akpinar, E. K., Bicer, Y., \& Cetinkaya, F. (2006). Modelling of thin layer drying of parsley leaves in a convective dryer and under open sun. Journal of Food Engineering 75(3), 308-315. https://doi.org/10.1016/j.jfoodeng.2005.04.018

Alezandro, M. R., Granato, D., \& Genovese, M. I. (2013). Jaboticaba (Myrciaria jaboticaba (Vell.) Berg), a Brazilian grape-like fruit, improves plasma lipid profile in streptozotocin-mediated oxidative stress in diabetic rats. Food Research International, 54(1), 650-659. https://doi.org/10.1016/j.foodres.2013.07.041

Almeida, R. L. J., Santos, N. C., Pereira, T. S., Barros, E. R., \& Cabral, M. B., et al. (2020) Análise granulométrica diferencial da casca de jabuticaba (Mirciaria cauliflora Berg). Research, Society and Development, 9(1), e156911875. http://dx.doi.org/10.33448/rsd-v9i1.1875

Ascheri, D. P. R., Ascheri, J. L. R., \& Carvalho, C. W. P. (2006). Caracterização da farinha de bagaço de jabuticaba e propriedades funcionais dos extrusados. Ciênc. Tecnol. Aliment., 26(4), 897-905. http://dx.doi.org/10.1590/S0101-20612006000400029

Alexandre, H. V., Gomes, J. P., Neto, A. L. B., Silva, F. L. H., \& Almeida, F. A. C. (2009). Cinética de secagem de abacaxi cv pérola em fatias. Revista Brasileira de Produtos Agroindustriais, 11(2), 123-128. 10.15871/1517-8595/rbpa.v11n2p123-128

Araujo, W. D., Goneli, A. L. D., Corrêa, P. C., Filho, C. P. H., \& Martins, E. A. S. (2017). Modelagem matemática da secagem dos frutos de amendoim em camada delgada. Revista Ciência Agronômica, 48(3), 448-457.

Brunini, M. A., Oliveira, A. L., Salandini, C. A. R., \& Bazzo, F. R. (2004). Influência de embalagens e temperatura no armazenamento de jabuticabas (Myrciaria jabuticaba (Vell) Berg) cv 'Sabará'. Ciênc. Tecnol. Aliment, 24(3), 378-383. https://doi.org/10.1590/S0101-20612004000300013.

Cipriano, P. A. (2011). Antocianinas de açaí (euterpe oleracea mart.) e casca de jabuticaba (myrciaria jaboticaba) na formulação de bebidas. Dissertação de Mestrado em Ciência e Tecnologia de Alimentos, Universidade Federal de Viçosa.

Citadin, I., Danner, M. A., Sasso, S. A. Z. (2010). Jabuticabeiras. Revista Brasileira de Fruticultura, 2(1), 2-16.

Dionello, R. G., Berbert, P. A., Molina, M. A. B., Pereira, R. C., \& Viana, A. P. et al. (2009). Secagem de fatias de abacaxi in natura e pré-desidratadas por imersão-impregnação: cinética e avaliação de modelos. Ciência e Tecnologia de Alimentos, 29(1), 232-240. https://doi.org/10.1590/S010120612009000100036.

Donadio, L. C. Jabuticaba (Myrciaria Jabuticaba (Vell.) Berg). FUNEP, 25.

Farias, R. Q, Texeira, I. R., Devilla, I. A., Ascheri, D. P. R \& Resende, O. (2012). Cinética de secagem de sementes de crambe, Revista Brasileira de Engenharia Agrícola e Ambiental, 16(5), 573-583. https://doi.org/10.1590/S1415-43662012000500014

Ferreira, A. E., Ferreira, B. S., Lages, M. M. B., Rodrigues, V. A. F, Thé, P. M. P. et al. (2012). Produção, caracterização e utilização da farinha de casca de jabuticaba em biscoitos tipo cookie. Alim. Nutr., 23(4), 603-607. http://serv-bib.fcfar.unesp.br/seer/index.php/alimentos

Fioreze, R. (2004). Principios de secagem de produtos biologicos, João Pessoa. Editora Universitária, p.229.

Garcia, L. G., Vendruscolo, F., Silva, F. A., Asquieri, E. R., \& Damiani, C. (2020) Casca de jabuticaba cristalizada: uma nova estratégia para industrialização de subprodutos. Research, Society and Development, 9(5), e27953158. http://dx.doi.org/10.33448/rsd-v9i5.3158

Gouveia, J. P. G., Almeida, F. A. C., Farias, E. S., Silva, M. M., Chaves, M. C. V et al. (2003). Determinação das curvas de secagem em frutos de cajá. Revista Brasileira de Produtos Agroindustriais, Especial (1), 65-68.

Gurak, P. D., De Bona, G. S., Tessaro, I. C., \& Marczack, L. D. F. (2014). Jaboticaba pomace powder obtained as a co-product of Juice extraction: A comparative study of powder obtained from peel and whole fruit. Food Research International, 62, 786-792. https://doi.org/10.1016/j.foodres.2014.04.042

Janjai, S., Mahayothee, B., Lamlert, N., Bala, B. K., Precoppe, M. et al. (2010). Diffusivity, shrinkage and simulated drying of litchi fruit (Litchi chinensis Sonn.). Journal of Food Engineering, 96(1), 214-221. https://doi.org/10.1016/j.jfoodeng.2009.07.015

Lima, A. J. B., Corrêa, A. D., Alves, A. P. C., Abreu, C. M. P., \& Dantas-Barros, A. M. (2008). Chemical characterization of the jabuticaba fruits (Myrciaria caulifloraBerg) and their fractions. Archivos Latinoamericanos de Nutrición, 58(4), 416-421.

Machado, A. V., Souza, J. A., \& Novaes, R. S. (2015). Estudo cinético da secagem da uva Isabel para produção de uva passa. Revista Verde de Agroecologia e Desenvolvimento Sustentável, 10(1), 47 - 51. 10.18378/rvads.v10i1.3324 
Machado, A.V., Barbosa, L.S, Macedo, J. L., \& Santos, C. M. (2014). Estudo da secagem de frutos tropicais do Nordeste. Revista Verde de Agroecologia e Desenvolvimento Sustentável, 9(1), 186-190. https://www.gvaa.com.br/revista/index.php/RVADS/article/view/2702

Manica, I. (2000). Frutas nativas, silvestres e exóticas: Técnicas de produção e mercado: abiu, aroma-preta, araçá, bacuri, biribá, carambola, cereja-do-riogrande, jabuticaba. Porto Alegre: Cinco continentes, 372p.

Marsiglia, W. I. M. L., Santiago, A. M., Alves, H. G., Almeida, R. L. J., Santos, N. C. et al. (2021) Modelagem e simulação do processo de secagem das cascas de jabuticaba (Myrciaria cauliflora). Research, Society and Development, 10(3), e21510313214. http://dx.doi.org/10.33448/rsd-v10i3.13214

Medeiros, J. (2007). Secagem e armazenamento de polpa de mangaba. Dissertação de Mestrado (Engenharia Agrícola) - PPGEA- Universidade Federal de Campina Grande, Campina Grande.

Nascimento, A. P. S., Barros, S. L., Santos, N. C., Araújo, A. J. B., Cavalcanti, A. S. R. R. M. et al. (2018). Secagem convectiva e influência da temperatura nas propriedades físico-químicas das amêndoas de girassol comercial. Revista Brasileira de Produtos Agroindustriais, 20(3), $227-238$.

Nunes, J. S., Castro, D. S., Moreira, I. S., Sousa, F. C. \& Silva, W. P. (2014). Descrição cinética de secagem da polpa de jabuticaba usando modelos empíricos. Revista Verde de Agroecologia e Desenvolvimento Sustentável. 2014

Reis, R. C., Barbosa, L.S., Lima, M. L., Reis, J. S., Devilla, I. A. et al. (2011). Modelagem matemática da secagem da pimenta Cumari do Pará. Revista Agrícola e Ambiental, 15(1), 347-353. https://doi.org/10.1590/S1415-43662011000400003

Santiago, A. M., Sousa, C. A. B., Segundo, V. A. G., Alcântara, S. R. \& Conrado, L. S. O. Aproveitamento da casca desidratada de jabuticaba para obtenção de poligalaturonase por fermentação em estado sólido. In: XIX Congresso Brasileiro de engenharia Química, 2012, CD-Rom.

Santos, J. A. B., Silva, G. F. \& Paganis, A. A. C. (2012). Estudo da cinética de secagem da pimenta malagueta (capsicum spp) cultivada no estado de Sergipe. Revista GEINTEC, 2(5), 465-471.

Silva, B. M., Silva, G. C. O., Saouza, A. H., Gonçalves, A. C. A., \& Carlos, L. A (2020) Elaboração de creme de jabuticaba como estratégia para aproveitamento total do fruto: caracterização físico-química e avaliação de compostos bioativos. Research, Society and Development, $9(8)$, e617986097. http://dx.doi.org/10.33448/rsd-v9i8.6097

Silva, M. C. (2012). Aproveitamento do resíduo do despolpamento da Jaboticaba (Myrciaria cauliflora Berg). Dissertação de Mestrado (Engenharia de Alimentos) -PPGEA- Universidade de São Paulo, Pirassununga.

Silva, J. N., Pinheiro Filho, J. B., Sediyama, G. C., \& Condé, A. R. (1979) Curvas de equilíbrio higroscópico do cacau. Revista Brasileira de Armazenamento, 4(1), 31-38.

Silva, G. J. F. D., Constant, P. B. L., Figueiredo, R. W. D., \& Moura, S. M. (2010). Formulation and stability of anthocyanins's colorants formulated with peels jabuticaba (Myrciariassp.). Alimentos e Nutrição, 21(3), 429-436.

Silva, V. M. A, Almeida, R. L. J., Santos, N. C., Muniz, C. E. S., Barros, E. R. et al. (2020) Predição de modelos matemáticos sobre a cinética de secagem de nozes (Juglans regia L.) sem casca. Research, Society and Development, 9(7), e522974390. http://dx.doi.org/10.33448/rsd-v9i7.4390

Sousa, B. A. A., Correia, R. T. P. (2010). Biotechmological reuse of fruit residues as rational strategy for agro-industrial resources. Journal of Technology Management \& Innovation, 5(2), 1-9. http://dx.doi.org/10.4067/S0718-27242010000200010

Texeira, N. C. (2011). Desenvolvimento, caracterização físico-química e avaliação sensorial de suco de jabuticaba (myrciaria jaboticaba (vell) berg), Dissertação de Mestrado (Ciências de Alimentos) - PPGEA - Universidade Federal de Minas Gerais.

Tosta, M. F., Salvio, L. G. A., Côrrea, J. L. G., \& Andrade, E. T. (2020) Modelagem matemática da cinética de secagem do café (Coffea arábica L.). Research, Society and Development, 9(7), e908974359. http://dx.doi.org/10.33448/rsd-v9i7.4359 